\title{
STABILITY OF PLANE WAVE SOLUTIONS OF COMPLEX GINZBURG-LANDAU EQUATIONS
}

BY

\section{B. J. MATKOWSKY AND V. VOLPERT}

Northwestern University, Evanston, Illinois

\begin{abstract}
We consider the stability of plane wave solutions of both single and coupled complex Ginzburg-Landau equations and determine stability domains in the space of coefficients of the equations.
\end{abstract}

1. Introduction. Ginzburg-Landau equations, both real and complex, arise in many problems of physical interest. First derived as long wave amplitude equations by Newell and Whitehead [12] and Segel [20], real Ginzburg-Landau equations describe, for example, convection in binary mixtures near the onset of instability, or flames stabilized on a burner [16]. Complex Ginzburg-Landau (CGL) equations were derived by Stewartson and Stuart [23] who studied plane Poiseuille flows, by Ermentrout [2] who extended the results of Howard and Kopell [3] on $\lambda-\omega$ systems to general reaction diffusion systems, and by others (see also $[6,11]$ and the references therein).

Actually, CGL equations describe the evolution of the amplitudes of unstable modes for any process exhibiting Hopf bifurcation, for which the continuous band of unstable wave numbers is taken into account. Therefore, the equations have become a self-significant object of study.

Coupled CGL equations govern the amplitudes, on slow spatial and temporal scales, of traveling waves propagating in opposite directions on the faster temporal and spatial scales associated with the original problem from which the GinzburgLandau equations were derived. The case when one of the amplitudes equals zero corresponds to a traveling wave solution of the original problem, while the case when the moduli of the two amplitudes are equal corresponds to a standing wave.

The simplest solutions of CGL equations are plane wave solutions and the present paper is devoted to an analysis of their stability. Stability of traveling wave solutions of a single CGL equation, which arises when the most unstable wave number is $k=0$, was studied by Eckhaus [1], Lange and Newell [8], Stuart and DiPrima [24], and Ermentrout [2] (see also [6] and the references therein) and others. Here we give more complete results for this case (Sec. 2). In addition we consider the case $k \neq 0$, for which, due to reflection symmetry, there are two amplitudes of waves

Received June 13, 1991.

Key words and phrases. Plane waves, stability, Ginzburg-Landau equations.

This work was supported in part by D.O.E. Grant DE-FG02-87ER25027 and N.S.F. Grant CTS 9008624.

(C) 1993 Brown University 
propagating in opposite directions, whose evolution as functions of slow temporal and spatial variables is described by coupled CGL equations. The stability of both traveling wave and standing wave solutions of coupled CGL equations is studied in Sec. 3.

We consider the case that the real parts of the coefficients of the diffusive terms are positive, and the real parts of certain nonlinear terms are negative; otherwise, plane wave solutions are necessarily unstable. The equations are scaled so that these terms are +1 and -1 respectively. Interesting results for the case that plane wave solutions are unstable, were obtained by Schöpf and Kramer [19], Sirovich et al [13, $14,18,21,22]$, Keefe [5], Kuramoto and Koga [7], Moon et al [10], Nozaki and Bekki [15], and others.

2. Stability of traveling wave solutions of a single CGL equation. We consider the scaled complex Ginzburg-Landau equation

$$
\frac{\partial R}{\partial t}=R+(1+i a) \frac{\partial^{2} R}{\partial x^{2}}+(-1+i b) R|R|^{2}
$$

where $a$ and $b$ are real constants.

We seek solutions in the form of traveling waves

$$
R=\rho e^{i(\omega t+k x)},
$$

where $\rho \geq 0$, and $\omega$ and $k$ are real constants. In addition to the trivial solution $\rho=0$, we obtain the one-parameter family of solutions

$$
\rho^{2}=1-k^{2}, \quad \omega=b-(a+b) k^{2} .
$$

To study the stability of the traveling wave solutions we represent $R$ as

$$
R=(\rho+r) e^{i(\omega t+k x)} .
$$

Substituting (2.4) into (2.1) and linearizing we obtain

$$
\frac{\partial r}{\partial t}=(1+i a)\left(2 i k \frac{\partial r}{\partial x}+\frac{\partial^{2} r}{\partial x^{2}}\right)+(-1+i b) \rho^{2}(r+\bar{r})
$$

We set $r=u+i v$ and seek normal mode solutions of the form

$$
u=e^{\Omega t+i \kappa x} \tilde{u}, \quad v=e^{\Omega t+i \kappa x} \tilde{v},
$$

where $\tilde{u}$ and $\tilde{v}$ are constants. Substituting (2.6) into (2.5) we find that nontrivial solutions exist for two values of $\Omega$ determined by the dispersion relation

$$
\Omega^{2}-2 p \Omega+q=0,
$$

where

$$
\begin{gathered}
p_{r}=-\kappa^{2}-\rho^{2}, \quad p_{i}=-2 \kappa k a, \\
q_{r}=\kappa^{4}\left(1+a^{2}\right)-4 \kappa^{2} k^{2}\left(1+a^{2}\right)-2 \rho^{2} \kappa^{2}(-1+a b), \\
q_{i}=4 \kappa k \rho^{2}(a+b) .
\end{gathered}
$$


Here the subscripts $r$ and $i$ denote the real and imaginary parts of the indicated quantity. In order for the plane wave solution to be stable we must have $\Re\left(\Omega_{j}\right)<0$ $(j=1,2)$, which is equivalent to

$$
4 p_{r}^{2} q_{r}+4 p_{r} p_{i} q_{i}>q_{i}^{2}
$$

Substituting (2.8)-(2.10) into (2.11) we obtain

$$
a_{1} \kappa^{8}+a_{2} \kappa^{6}+a_{3} \kappa^{4}+a_{4} \kappa^{2}>0
$$

where

$$
\begin{aligned}
& a_{1}=1+a^{2}, \\
& a_{2}=2 \rho^{2}\left(1+a^{2}\right)-4 k^{2}\left(1+a^{2}\right)-2 \rho^{2}(-1+a b), \\
& a_{3}=\rho^{4}\left(1+a^{2}-4(-1+a b)\right)+8 \rho^{2} k^{2}(-1+a b), \\
& a_{4}=-2 \rho^{4}\left(\rho^{2}(-1+a b)+2 k^{2}\left(1+b^{2}\right)\right) .
\end{aligned}
$$

The traveling wave solution (2.2)-(2.3) will be stable if and only if the inequality (2.12) is satisfied for all values of $\kappa$. We will derive necessary and sufficient conditions on the coefficients $a$ and $b$ as well as the wave number $k$ for the inequality to be satisfied. To do so we introduce the parameter $\alpha \geq 0$ defined by $k^{2}=\alpha \rho^{2}$. Then taking (2.3) into account, we have

$$
k^{2}=\frac{\alpha}{1+\alpha}, \quad \rho^{2}=\frac{1}{1+\alpha} .
$$

Introducing

$$
\xi=\frac{-1+a b}{1+a^{2}}, \quad \eta=\frac{1+b^{2}}{1+a^{2}}, \quad y=\frac{\kappa^{2}}{1+\alpha},
$$

we divide (2.12) by $\kappa^{2}$ and rewrite inequality (2.12) as

$$
f(y)=y^{3}+2 y^{2}(1-2 \alpha-\xi)+y(1-4 \xi+8 \alpha \xi)-2 \xi-4 \alpha \eta>0,
$$

which must be satisfied for all positive $y$ in order that the traveling wave solution (2.2)-(2.3) be stable.

An obvious necessary condition is

$$
\xi+2 \alpha \eta<0,
$$

which can be written in terms of the original parameters $a$ and $b$ as

$$
2 \alpha\left(1+b^{2}\right)+a b<1 .
$$

If $\alpha=0$, which corresponds to $k=0,(2.16)$ reduces to

$$
a b<1 \text {, }
$$

which describes the stability region for the Stokes solution, which is a spatially uniform, time periodic solution of (2.1), as discussed, e.g., in [21].

In regions of the $(b, a)$ plane determined by $(2.16)$, traveling wave solutions are stable to long-wave $(\mathrm{LW})(\kappa \ll 1)$ perturbations. The regions are shown in Fig. 1 for different values of $\alpha$ (see pp. 268 and 269). 


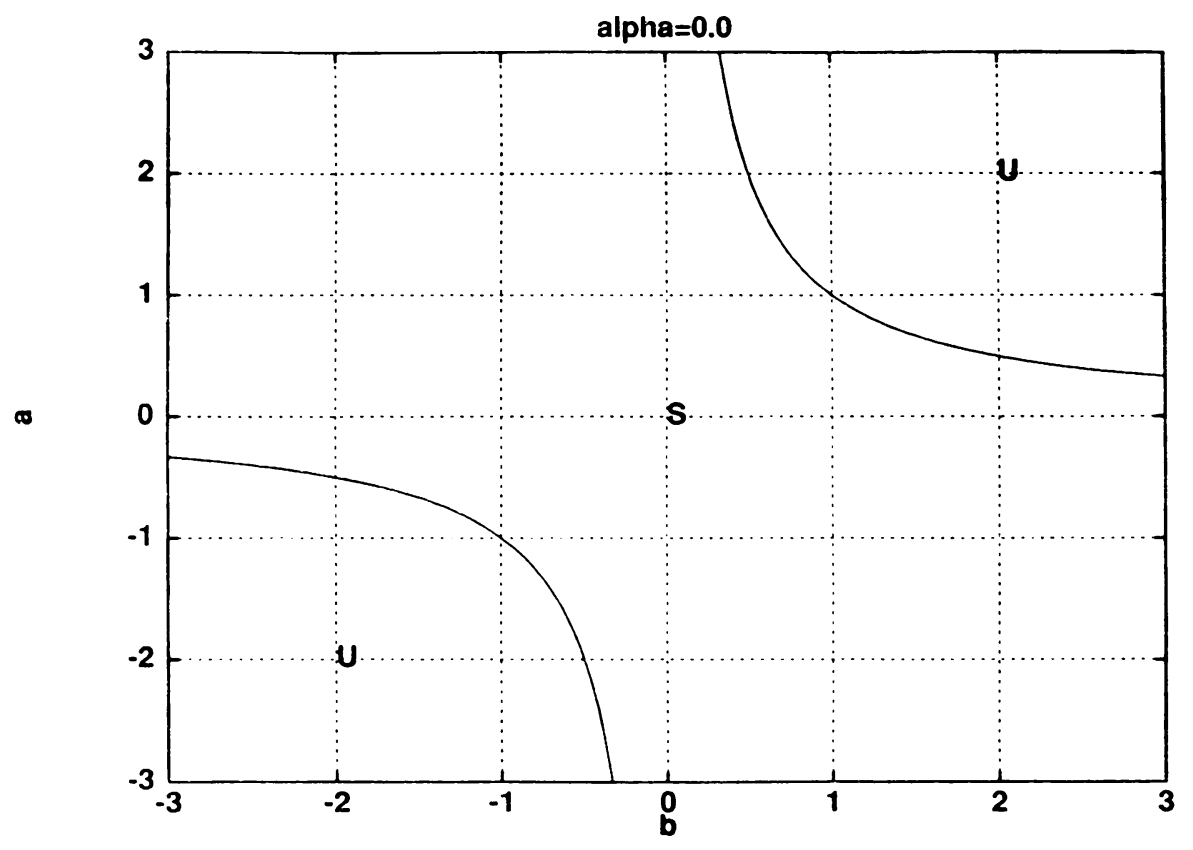

FIG. 1(a). Region of LW stability in the $(b, a)$ plane for a single complex Ginzburg-Landau equation; $\alpha=0$.

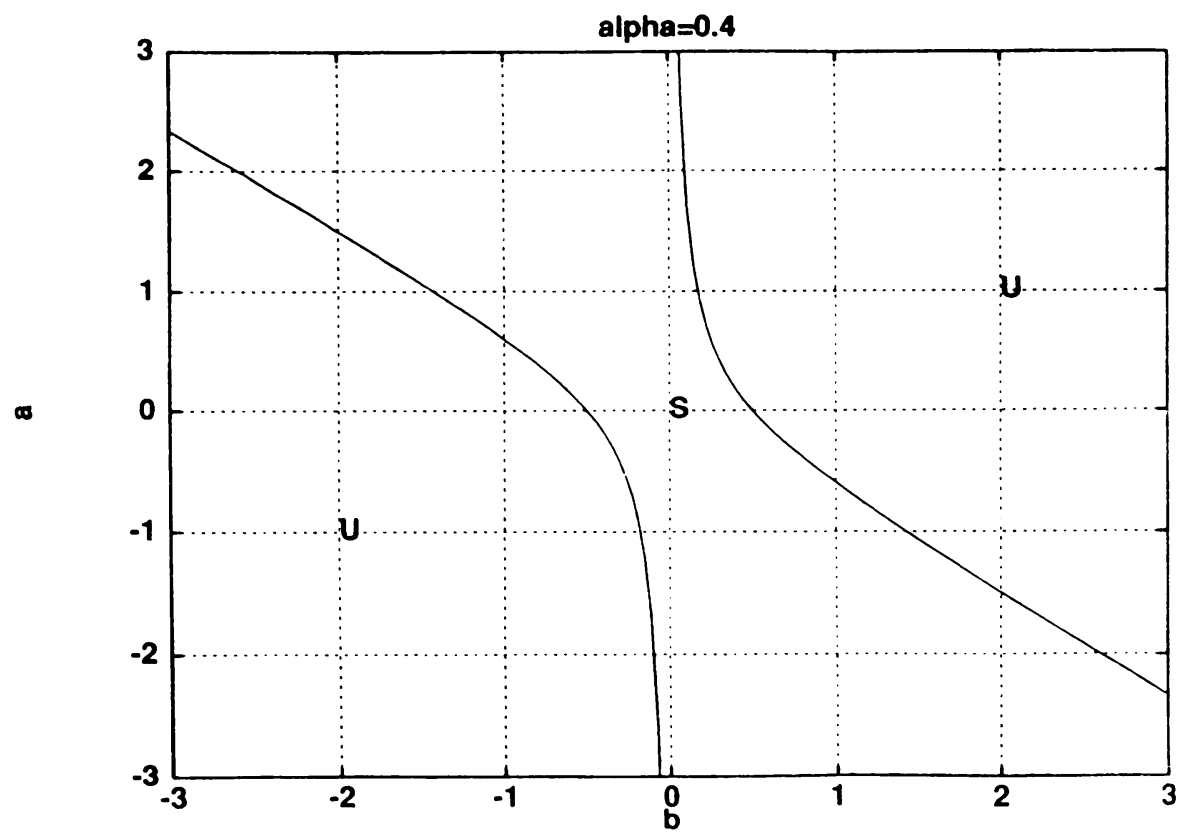

FIG. 1(b). Region of LW stability in the $(b, a)$ plane for a single complex Ginzburg-Landau equation; $\alpha=0.4$. 


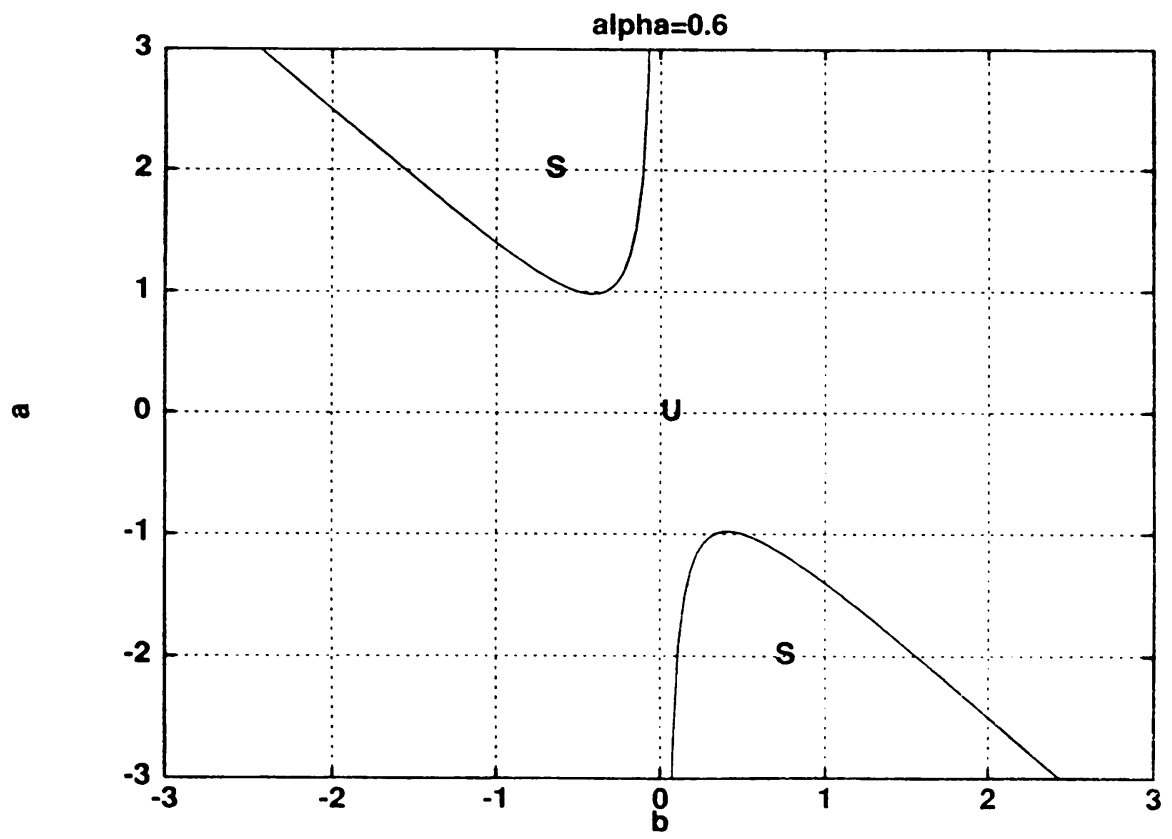

Fig. 1(c). Region of LW stability in the $(b, a)$ plane for a single complex Ginzburg-Landau equation; $\alpha=0.6$.

It is clear that condition (2.16) is not sufficient for (2.15) to be satisfied. We now give a brief derivation of sufficient conditions. We consider the critical case which corresponds to the stability boundary in the space of parameters, when for some value of $y$, say $y_{*}, f(y)$ has a minimum that is tangent to the axis $f=0$, i.e.,

$$
f\left(y_{*}\right)=0, \quad f^{\prime}\left(y_{*}\right)=0,
$$

assuming that $(2.16)$ is satisfied. Solving these two equations simultaneously yields both the value of $y_{*}$ as a function of $a, b$, and $\alpha$ and the relation between $a$ and $b$ at the stability boundary. Actually it is more convenient to determine $a$ and $b$ (equivalently, $\xi$ and $\eta$ ) as functions of $y_{*}$ to obtain (we omit the subscript)

$$
\begin{gathered}
\xi(y)=\frac{3 y^{2}+4 y(1-2 \alpha)+1}{4(y+1-2 \alpha)}, \\
\eta(y)=\frac{1}{4 \alpha}\left(y^{3}+2 y^{2}(1-2 \alpha-\xi(y))+y(1-4 \xi(y)(1-2 \alpha))-2 \xi(y)\right) .
\end{gathered}
$$

Taking into account $(2.14)$ we obtain

$$
\begin{gathered}
b(y)= \pm \sqrt{\frac{(\xi(y)+\eta(y))^{2}}{\eta(y)-\xi^{2}(y)}}, \\
a(y)=\frac{1}{b(y)}\left(\frac{\xi(y)}{\eta(y)}\left(1+b^{2}(y)\right)+1\right) .
\end{gathered}
$$

Formulas (2.17)-(2.20) provide a parametric representation of the stability boundaries with respect to perturbations with finite wave (FW) numbers in the $(b, a)$ 
plane. The range of variation of the parameter $y$ in these formulas is determined by the conditions

$$
\begin{gathered}
\xi(y)+2 \alpha \eta(y) \leq 0, \\
\eta(y)>\xi^{2}(y) .
\end{gathered}
$$

The former condition corresponds to $f(0) \geq 0$, and the latter condition to the requirement that $b$ is real. It can be shown that the conditions $(2.21),(2.22)$ cannot be satisfied when $\alpha<\frac{2}{3}$ or $\alpha>1$. However, for all points $(b, a)$ lying inside the stability regions $\mathrm{S}$ (see Fig. 1), if $\alpha<\frac{2}{3}(\alpha>1)$, the traveling wave solution (2.2) is stable (unstable) with respect to FW perturbations. For $\frac{2}{3}<\alpha<1$, there exists a range of $y$ satisfying (2.21)-(2.22). Therefore, there exist FW stability boundaries inside the $\mathrm{LW}$ stability region, that is, $\mathrm{S}$ is subdivided into two subregions inside which the traveling wave solutions are respectively stable and unstable to FW perturbations. These are exhibited in Fig. 3 at the end of this paper. This range of variation of $y$ lies in the interval $(0,1)$ and is given by (see Fig. 2)

$$
\begin{array}{cc}
0<y<y^{*}(\alpha) & \left(\frac{2}{3}<\alpha<\frac{3}{4}\right), \\
4 \alpha-3<y<y^{*}(\alpha) & \left(\frac{3}{4}<\alpha<1\right),
\end{array}
$$

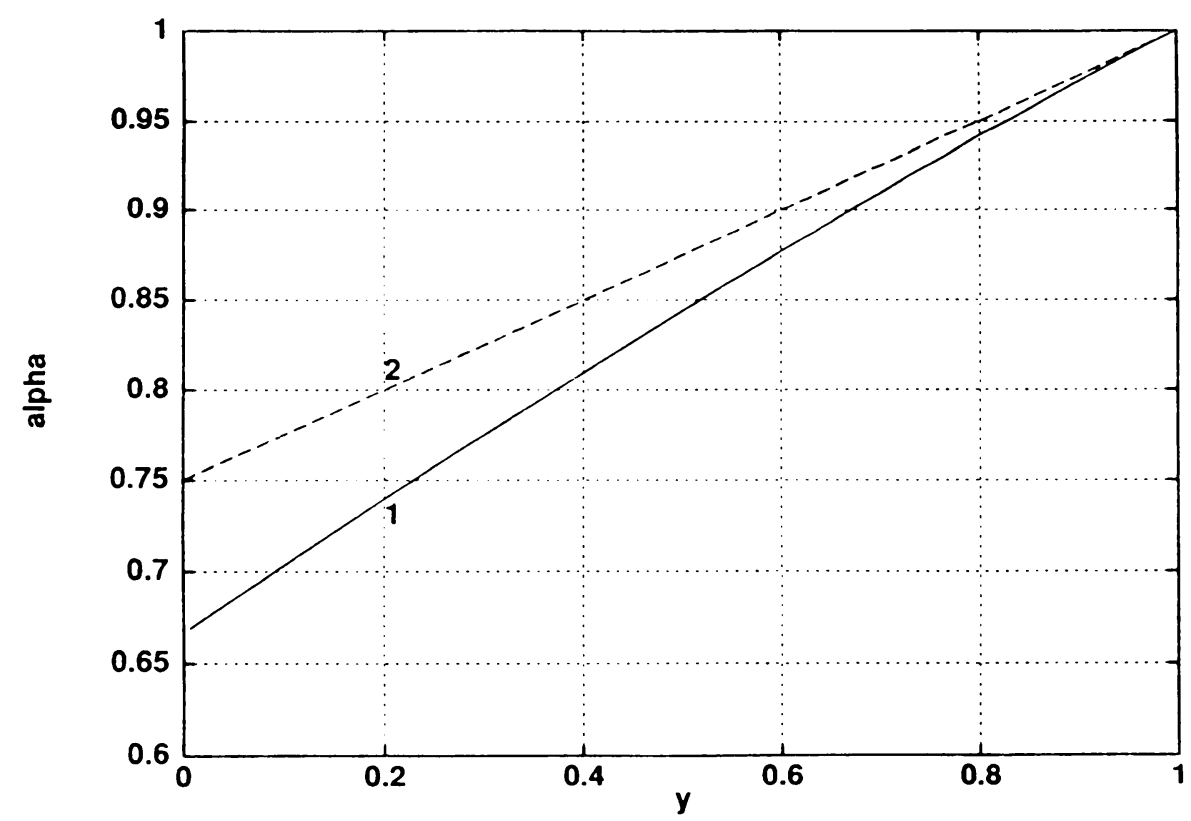

FIG. 2. Ranges of variation of $y$ in the parametric dependence (2.17)-(2.20). For a fixed $\alpha\left(\frac{2}{3}<\alpha<\frac{3}{4}\right), y$ varies between zero and $y^{*}($ see $(2.23))$, and between $4 \alpha-3$ and $y^{*}$ for $\frac{3}{4}<\alpha<1$. 
where $y^{*}(\alpha)$ is the inverse function of

$$
\alpha(y)=\frac{1}{2}+\frac{4 y^{3}+y^{2}+2 y+1}{11 y^{2}+2 y+3+\sqrt{(1-y)^{3}(7 y+9)}} .
$$

Stability regions in the $(b, a)$ plane are shown in Fig. 3 for different values of $\alpha$. Points $(b, a)$ lying in the regions $\mathrm{S}, \mathrm{U}$, and $\mathrm{U} 1$ correspond to traveling wave solutions that are stable, and unstable to $\mathrm{LW}$ and FW perturbations, respectively.

Let us consider the trajectory in the $(b, a)$ plane, of the point of intersection of the LW and FW stability boundaries as $\alpha$ varies from $\frac{2}{3}$ to 1 . Substituting $y=0$ for $\frac{2}{3}<\alpha<\frac{3}{4}$ and $y=4 \alpha-3$ for $\frac{3}{4}<\alpha<1$ into (2.19)-(2.22), we obtain

$$
\begin{gathered}
a=\frac{-2 b\left(b^{2}-1\right)+\sqrt{4 b^{2}\left(b^{2}-1\right)^{2}+\left(3 b^{2}-1\right)\left(5 b^{2}+1\right)}}{3 b^{2}-1} \quad\left(b^{2}>\frac{1}{3}\right), \\
a=-\frac{1}{b} \quad\left(b^{2}<\frac{1}{3}\right) .
\end{gathered}
$$

The curves are shown with dashed lines in Fig. 4 at the end of this paper. The solid line in this figure is the curve

$$
a=\frac{1}{b} .
$$

These curves divide the $(b, a)$ plane into 5 regions. The stability results for traveling wave solutions of a single Ginzburg-Landau equation are as follows. For each pair of coefficients $b, a$ lying in the regions $\mathrm{U}$, traveling waves with any wave number $k$ are unstable. If the coefficients $b, a$ lie in the region $\mathrm{S} 1$, there is a critical wave number $k_{\mathrm{cr}}$,

$$
k_{\mathrm{cr}}^{2}=\frac{1-a b}{3+2 b^{2}-a b},
$$

such that traveling waves with $k<(>) k_{\mathrm{cr}}$ are stable (unstable). The loss of stability when $k=k_{\mathrm{cr}}$ is due to LW perturbations. If the coefficients $b, a$ lie in one of the regions $\mathrm{S} 2$, then there exists a second critical value of $k$, say, $k_{*}=k_{*}(a, b)$ such that the traveling wave solutions are stable when $k<k_{*}$ and unstable otherwise. The quantity $k_{*}^{2}$ varies from $\frac{2}{5}$ to $\frac{1}{2}$ for $b, a$ lying in S2. The loss of stability when $k=k_{*}$ is due to $\mathrm{FW}$ perturbations. The value $k_{*}(a, b)$ can be found explicitly, though we do not present it because the expression is awkward. To find $k_{*}(a, b)$ we simply solve (2.17) for $\alpha$, substitute the result into (2.18), solve the resulting equation for $y$, and thus find $\alpha$ as a function of $a$ and $b$. $\frac{1}{2}$.

We observe, in particular, that traveling wave solutions cannot be stable for $k^{2}>$

3. Stability of plane wave solutions of coupled complex Ginzburg-Landau equations. Let us now consider the system of coupled complex Ginzburg-Landau equations of 
the form

$$
\begin{aligned}
& \frac{\partial R}{\partial t}=R+(1+i a) \frac{\partial^{2} R}{\partial x^{2}}+(-1+i b) R|R|^{2}+c R|S|^{2} \\
& \frac{\partial S}{\partial t}=S+(1+i a) \frac{\partial^{2} S}{\partial x^{2}}+(-1+i b) S|S|^{2}+c S|R|^{2}
\end{aligned}
$$

Thus, we consider the case when the group velocity is so small that it is neglected at this order and only enters at higher order in the perturbation analysis which, at this order, yields (3.1)-(3.2). We note that if the group velocity is not small, the amplitude equations are nonlocal Ginzburg-Landau equations involving averaged terms [4, 9, 17]. We seek solutions of the form

$$
R=\rho_{1} e^{i\left(\omega_{1} t+k_{1} x\right)}, \quad S=\rho_{2} e^{i\left(\omega_{2} t+k_{2} x\right)},
$$

where $\rho_{j}, k_{j}$, and $\omega_{j}(j=1,2)$ are constants. In addition to the trivial solution $\rho_{1}=\rho_{2}=0$, we obtain the following nontrivial solutions:

$$
\begin{array}{ll}
\rho_{1}^{2}=1-k_{1}^{2}, \quad \rho_{2}=0, & \omega_{1}=b-(a+b) k_{1}^{2}, \\
\rho_{1}=0, \quad \rho_{2}^{2}=1-k_{2}^{2}, & \omega_{2}=b-(a+b) k_{2}^{2},
\end{array}
$$

and

$$
\begin{array}{ll}
\rho_{1}^{2}=\frac{1-k_{1}^{2}+c_{r}\left(1-k_{2}^{2}\right)}{1-c_{r}^{2}}, & \rho_{2}^{2}=\frac{1-k_{2}^{2}+c_{r}\left(1-k_{1}^{2}\right)}{1-c_{r}^{2}}, \\
\omega_{1}=-a k_{1}^{2}+b \rho_{1}^{2}+c_{i} \rho_{2}^{2}, & \omega_{2}=-a k_{2}^{2}+b \rho_{2}^{2}+c_{i} \rho_{1}^{2} .
\end{array}
$$

The single mode solutions (3.3) and (3.4) correspond to traveling wave solutions of the original problem, while the mixed mode solution (3.5) corresponds to a quasiperiodic wave in the original problem. If $k_{1}=k_{2}$, the solution (3.5) corresponds to a standing wave.

We consider infinitesimal perturbations $r_{1}$ and $r_{2}$ given by

$$
R=\left(\rho_{1}+r_{1}\right) e^{i\left(\omega_{1} t+k_{1} x\right)}, \quad S=\left(\rho_{2}+r_{2}\right) e^{i\left(\omega_{2} l+k_{2} x\right)} .
$$

Substituting into (3.1)-(3.2) and linearizing we obtain

$$
\begin{aligned}
& \frac{\partial r_{1}}{\partial t}=a_{11} r_{1}+(-1+i b) \rho_{1}^{2} \bar{r}_{1}+\rho_{1} \rho_{2} c\left(r_{2}+\bar{r}_{2}\right)+(1+i a)\left(2 i k_{1} \frac{\partial r_{1}}{\partial x}+\frac{\partial^{2} r_{1}}{\partial x^{2}}\right) \\
& \frac{\partial r_{2}}{\partial t}=a_{21} r_{2}+(-1+i b) \rho_{2}^{2} \bar{r}_{2}+\rho_{1} \rho_{2} c\left(r_{1}+\bar{r}_{1}\right)+(1+i a)\left(2 i k_{2} \frac{\partial r_{2}}{\partial x}+\frac{\partial^{2} r_{2}}{\partial x^{2}}\right)
\end{aligned}
$$

where the coefficients $a_{11}$ and $a_{21}$ are

$$
\begin{aligned}
& a_{11}=1+i \omega_{1}-(1+i a) k_{1}^{2}+2(-1+i b) \rho_{1}^{2}+c \rho_{2}^{2}, \\
& a_{21}=1+i \omega_{2}-(1+i a) k_{2}^{2}+c \rho_{1}^{2}+2(-1+i b) \rho_{2}^{2} .
\end{aligned}
$$

We first consider the stability of the traveling wave solutions. We treat the case (3.3) only, since the case (3.4) can be considered in a similar manner. We set $r_{j}=$ 
$u_{j}+i v_{j}(j=1,2)$ and look for normal mode solutions of the form

$$
\left(\begin{array}{c}
u_{j} \\
v_{j}
\end{array}\right)=e^{\Omega t+i \kappa x}\left(\begin{array}{c}
\tilde{u}_{j} \\
\tilde{v}_{j}
\end{array}\right),
$$

where $\tilde{u}_{j}$ and $\tilde{v}_{j}$ are constants. Substituting (3.8) into (3.6)-(3.7), we find that nontrivial solutions exist for four values of $\Omega$. Since for the single mode solutions the two equations (3.6)-(3.7) decouple, two of the values of $\Omega$ satisfy exactly the same equation (2.7) as for a single Ginzburg-Landau equation, while the other two satisfy

$$
\Omega^{2}-2 \hat{p} \Omega+\hat{q}=0,
$$

where

$$
\hat{p}=1+c_{r} \rho_{1}^{2}-\kappa^{2}, \quad \hat{q}=\hat{p}^{2}+\left(a \kappa^{2}-c_{i} \rho_{1}^{2}\right)^{2} .
$$

Therefore, in order for the traveling wave solution to be stable, we must have exactly the same conditions on the coefficients $a$ and $b$ and on the wave number $k_{1}\left(k_{1}^{2}=\alpha /(1+\alpha)\right)$ as for the single Ginzburg-Landau equation and, in addition,

$$
c_{r}<0, \quad k_{1}^{2}<1+\frac{1}{c_{r}} .
$$

These restrictions follow from the condition that the solutions of (3.9) must have negative real parts. We observe, in particular, that for the traveling wave solutions to be stable, it is necessary that

$$
c_{r}<-1 \text {. }
$$

We now proceed to study the stability of the standing wave solutions $\left(k_{1}=k_{2} \equiv k\right.$ in (3.5)). We observe from (3.5) that in this case $\rho_{1}=\rho_{2} \equiv \rho$ with

$$
\rho^{2}=\frac{1-k^{2}}{1-c_{r}} .
$$

We find that nontrivial solutions of (3.6)-(3.7) of the form (3.8) exist for four values of $\Omega$ determined by

$$
\Omega_{ \pm}^{2}-2 p^{ \pm} \Omega_{ \pm}+q^{ \pm}=0,
$$

where

$$
\begin{gathered}
p_{r}^{ \pm}=-\kappa^{2}+\left(-1 \pm c_{r}\right) \rho^{2}, \quad p_{i}^{ \pm}=-2 \kappa k a, \\
q_{r}=\kappa^{4}\left(1+a^{2}\right)-4 \kappa^{2} k^{2}\left(1+a^{2}\right)-2 \rho^{2} \kappa^{2}\left(\left(-1 \pm c_{r}\right)+a\left(b \pm c_{i}\right)\right), \\
q_{i}=4 \kappa k \rho^{2}\left(-a\left(-1 \pm c_{r}\right)+\left(b \pm c_{i}\right)\right) .
\end{gathered}
$$

In order for the standing wave to be stable, we must have, for all $\kappa$, that

$$
\begin{gathered}
p_{r}^{ \pm}<0, \\
4\left(p_{r}^{ \pm}\right)^{2} q_{r}^{ \pm}+4 p_{r}^{ \pm} p_{i}^{ \pm} q_{i}^{ \pm}>\left(q_{i}^{ \pm}\right)^{2} .
\end{gathered}
$$

The conditions (3.11) imply that

$$
-1<c_{r}<1,
$$


while the conditions (3.12) can be reduced to

$$
y_{ \pm}^{3}+2 y_{ \pm}^{2}\left(1-2 \alpha-\xi_{ \pm}\right)+y_{ \pm}\left(1-4 \xi_{ \pm}+8 \alpha \xi_{ \pm}\right)-2 \xi_{ \pm}-4 \alpha \eta_{ \pm}>0,
$$

where

$$
\begin{gathered}
\xi_{ \pm}=\frac{-1+a\left(\left(b \pm c_{i}\right) /\left(1 \mp c_{r}\right)\right)}{1+a^{2}} \\
\eta_{ \pm}=\frac{1+\left(b \pm c_{i}\right)^{2} /\left(1 \mp c_{r}\right)^{2}}{1+a^{2}} \\
y_{ \pm}=\frac{\kappa^{2}}{\rho^{2}\left(1 \mp c_{r}\right)} \\
\alpha=\frac{k^{2}}{1-k^{2}} .
\end{gathered}
$$

We observe that the left-hand side of (3.14) has exactly the same cubic form as in (2.14), where the coefficient $b$ in (2.16) is replaced by $\left(b \pm c_{i}\right) /\left(1 \mp c_{r}\right)$. Therefore, all the stability results for a traveling wave solution of a single Ginzburg-Landau equation, that we found in terms of the parameters $a$ and $b$, are valid for the stability of the standing wave solution of the coupled complex Ginzburg-Landau equations with the replacement of $b$ by $\left(b \pm c_{i}\right) /\left(1 \mp c_{r}\right)$, and with the additional restriction (3.13).

Comparing the necessary conditions (3.10) and (3.13) for traveling wave and standing wave solutions, respectively, to be stable, we observe that they cannot be stable simultaneously.

4. Summary. We considered a single Ginzburg-Landau equation

$$
\frac{\partial R}{\partial t}=R+(1+i a) \frac{\partial^{2} R}{\partial x^{2}}+(-1+i b) R|R|^{2}
$$

and studied the stability of traveling wave solutions

$$
\begin{gathered}
R=\rho e^{i(\omega t+k x)}, \\
\rho^{2}=1-k^{2}, \quad \omega=b-(a+b) k^{2} .
\end{gathered}
$$

The results are shown in Fig. 4. The dashed lines are given by

$$
\begin{gathered}
a=\frac{-2 b\left(b^{2}-1\right)+\sqrt{4 b^{2}\left(b^{2}-1\right)^{2}+\left(3 b^{2}-1\right)\left(5 b^{2}+1\right)}}{3 b^{2}-1} \quad\left(b^{2}>\frac{1}{3}\right), \\
a=-\frac{1}{b} \quad\left(b^{2}<\frac{1}{3}\right),
\end{gathered}
$$

and the solid line is the curve

$$
a=\frac{1}{b} .
$$

These curves divide the $(b, a)$ plane into 5 regions. For each point $b, a$ lying in a region $\mathrm{U}$, traveling waves with any wave number $k$ are unstable. In this region, 
chaotic behavior was observed numerically (see e.g. [6]). If the coefficients $b, a$ lie in the region $\mathrm{S} 1$, there is a critical wave number $k_{\mathrm{cr}}$,

$$
k^{2}<k_{\mathrm{cr}}^{2} \equiv \frac{1-a b}{3+2 b^{2}-a b},
$$

such that traveling waves with $k<(>) k_{\mathrm{cr}}$ are stable (unstable). The loss of stability when $k=k_{\mathrm{cr}}$ is due to LW perturbations $(\kappa \ll 1)$. If the coefficients $b, a$ lie in one of the regions $\mathbf{S} 2$, then there exists a critical value of $k$, say, $k_{*}=k_{*}(a, b)$ such that the traveling wave solutions are stable when $k<k_{*}$, and unstable otherwise. The quantity $k_{*}^{2}$ varies from $\frac{2}{5}$ to $\frac{1}{2}$ for $b, a$ lying in S2. The loss of stability when $k=k_{*}$ is due to $\mathrm{FW}$ perturbations. The value $k_{*}(a, b)$ can be found explicitly, though we do not present the formula because it is awkward.

We observe, in particular, that traveling wave solutions cannot be stable for $k^{2}>\frac{1}{2}$.

We also considered the stability of both traveling wave solutions

$$
\begin{gathered}
R=\rho e^{i(\omega t+k x)}, \quad S=0, \\
\rho^{2}=1-k^{2}, \quad \omega=b-(a+b) k^{2},
\end{gathered}
$$

and standing wave solutions

$$
\begin{gathered}
R=\rho e^{i(\omega t+k x)}, \quad S=\rho e^{i(\omega t+k x)}, \\
\rho^{2}=\frac{1-k^{2}}{1-c_{r}}, \quad \omega=-a k^{2}+b \rho^{2}+c_{i} \rho^{2},
\end{gathered}
$$

of coupled complex Ginzburg-Landau equations

$$
\begin{aligned}
& \frac{\partial R}{\partial t}=R+(1+i a) \frac{\partial^{2} R}{\partial x^{2}}+(-1+i b) R|R|^{2}+c R|S|^{2}, \\
& \frac{\partial S}{\partial t}=S+(1+i a) \frac{\partial^{2} S}{\partial x^{2}}+(-1+i b) S|S|^{2}+c S|R|^{2} .
\end{aligned}
$$

The results on the stability of traveling wave solutions are the same as for the single Ginzburg-Landau equation, with the additional restriction

$$
c_{r}<-1, \quad k^{2}<1+\frac{1}{c_{r}} .
$$

Stability results for the traveling wave solutions of the single Ginzburg-Landau equation, that we found in terms of the parameters $a$ and $b$, are also valid for the stability of the standing wave solution of the coupled complex Ginzburg-Landau equations, with the replacement of $b$ by $\left(b \pm c_{i}\right) /\left(1 \mp c_{r}\right)$ and the additional restriction

$$
-1<c_{r}<1 \text {. }
$$

It is clear from the above conditions that traveling wave and standing wave solutions cannot be stable simultaneously.

Though we did not explicitly study the stability of quasiperiodic waves, it is qualitatively clear that the results should be similar to those for the standing wave solutions. 
That is, the parameter space can be divided into subregions in which the solutions are stable and those in which they are unstable. The existence of regions of stability, at least for quasiperiodic waves corresponding to $k_{1}$ close to $k_{2}$, follows from that for standing wave solutions (for which $k_{1}=k_{2}$ ).

The only existing stability results for plane wave solutions of complex GinzburgLandau equations appear to be those for the single equation. Our results for scalar equations extend those in $[1,8,24]$. In [6] the stability of traveling wave solutions with respect to long-wavelength perturbations, that is small $\kappa$, is discussed. In fact, however, caution must be exercised in extrapolating the result for stability to LW perturbations to larger values of $\kappa$. While the stability boundaries in the two cases agree in parts of the $(b, a)$ plane, they can be shown to diverge from one another elsewhere. This is the case, e.g., when the leading term in (2.12) alone does not suffice to determine the stability boundary. In other words, when $\kappa \rightarrow 0$ the stability boundaries for FW perturbations converge to those for LW perturbations, but the limit is nonuniform with respect to $a$ and $b$. The result obtained in [6] for longwave stability corresponds to our condition (2.16). As we saw above, this condition is only necessary, but not sufficient to insure stability. Thus, for arbitrary $\kappa$, an additional stability boundary, not previously obtained, is determined by equations (2.17)-(2.20).

We are pleased to thank Professor A. G. Merzhanov and Dr. W. L. Grosshandler for helping to arrange the collaboration between the authors, under the auspices of the U.S.-USSR Program of Cooperation in Basic Scientific Research, jointly sponsored by the NSF and the USSR Academy of Sciences.

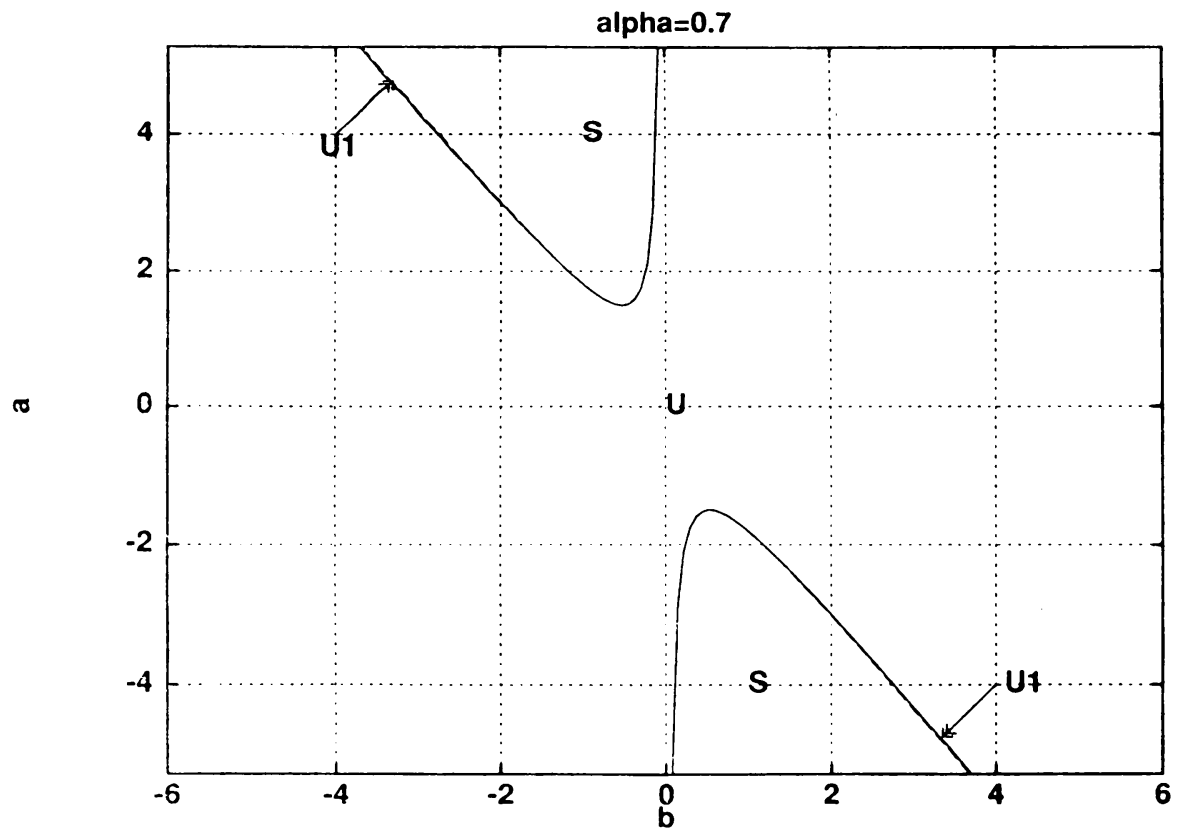

FIG. 3(a). Region of FW stability in the $(b, a)$ plane for a single complex Ginzburg-Landau equation; $\alpha=0.7$. 


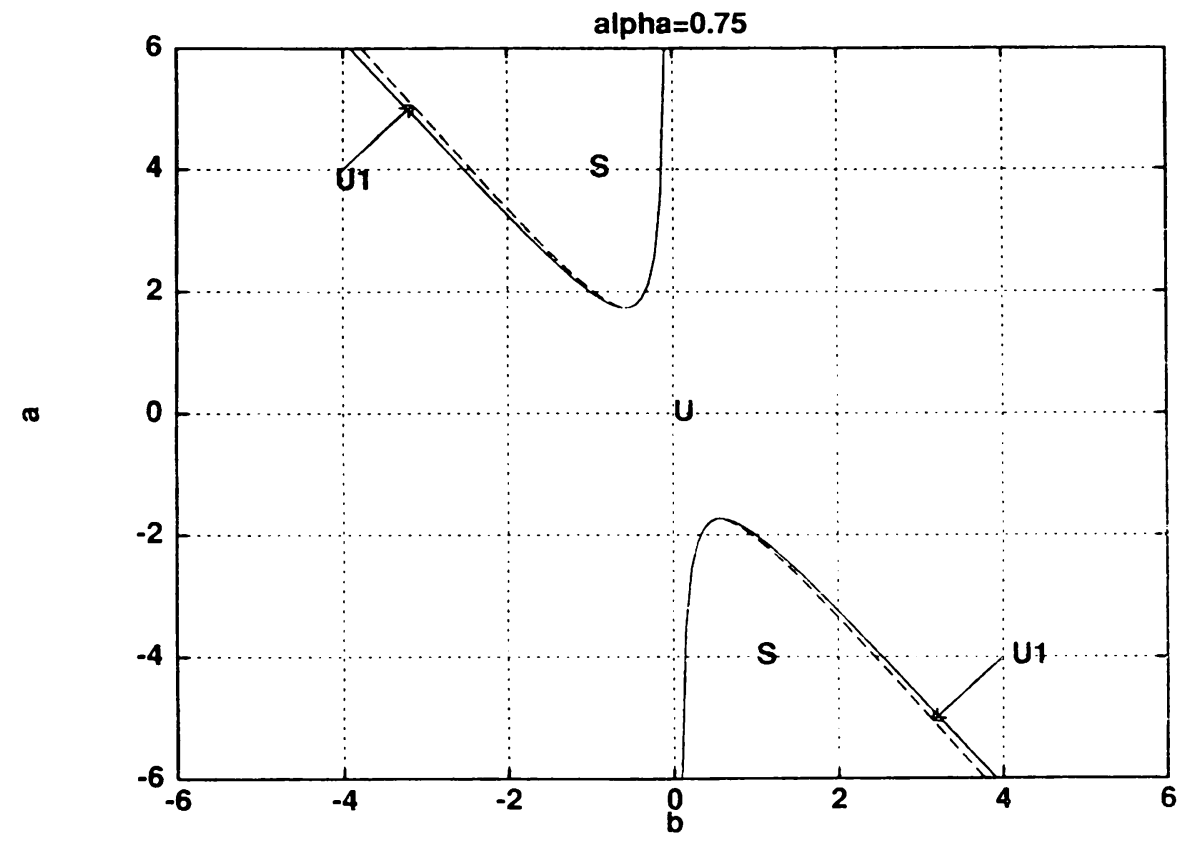

FIG. 3(b). Region of FW stability in the $(b, a)$ plane for a single complex Ginzburg-Landau equation; $\alpha=0.75$.

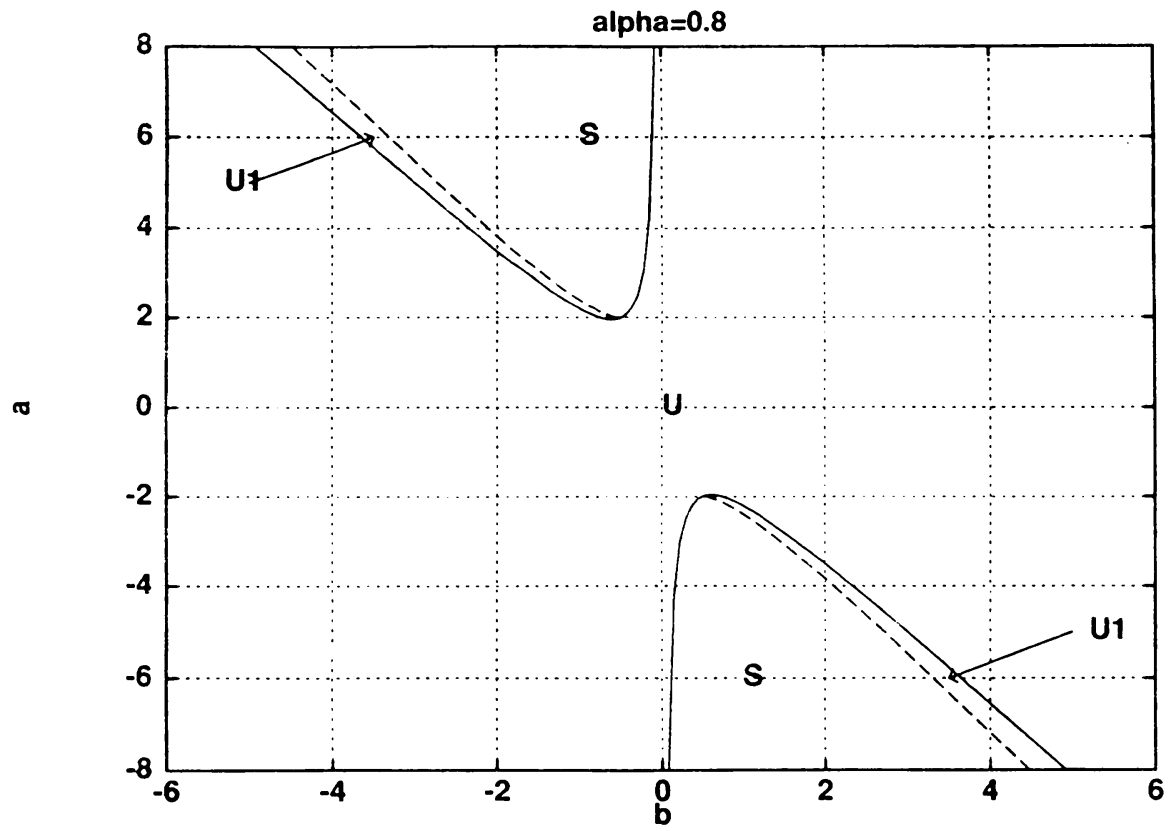

FIG. 3(c). Region of FW stability in the $(b, a)$ plane for a single complex Ginzburg-Landau equation; $\alpha=0.8$. 


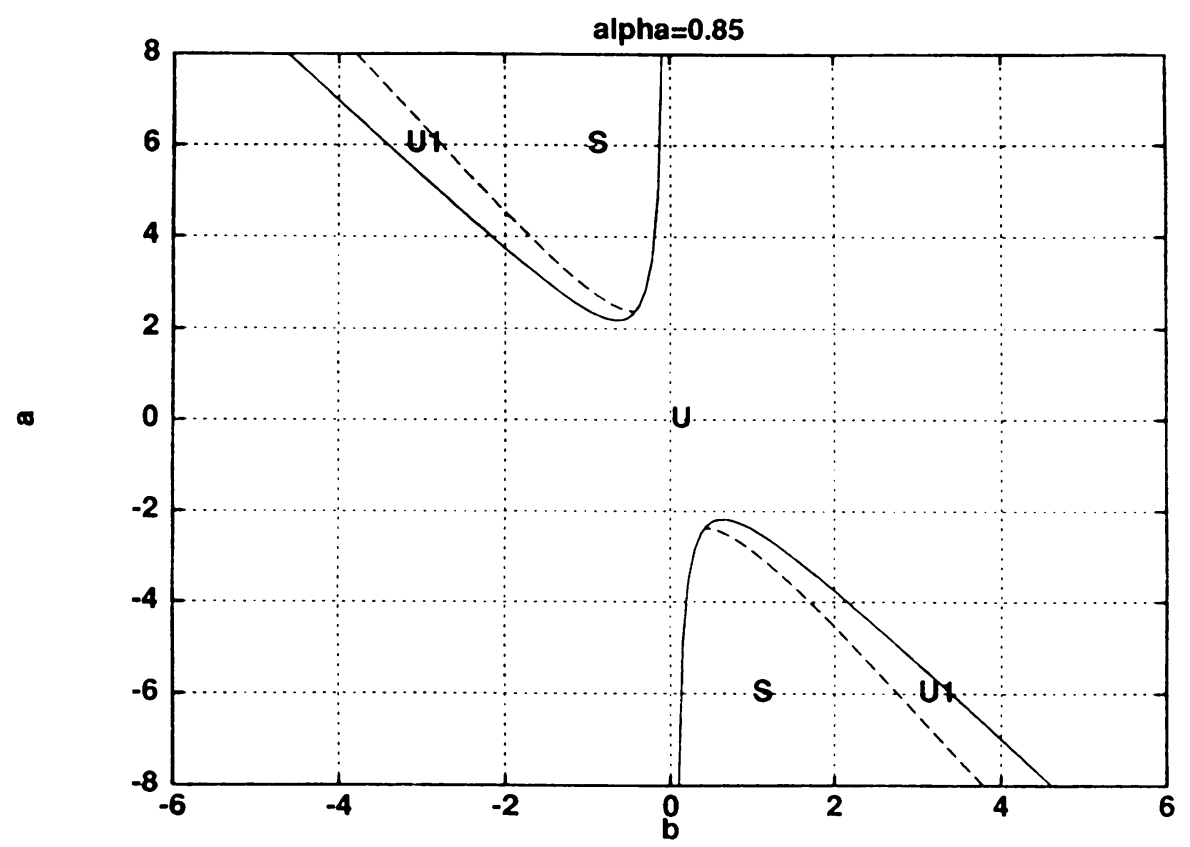

FIG. 3(d). Region of FW stability in the $(b, a)$ plane for a single complex Ginzburg-Landau equation; $\alpha=0.85$.

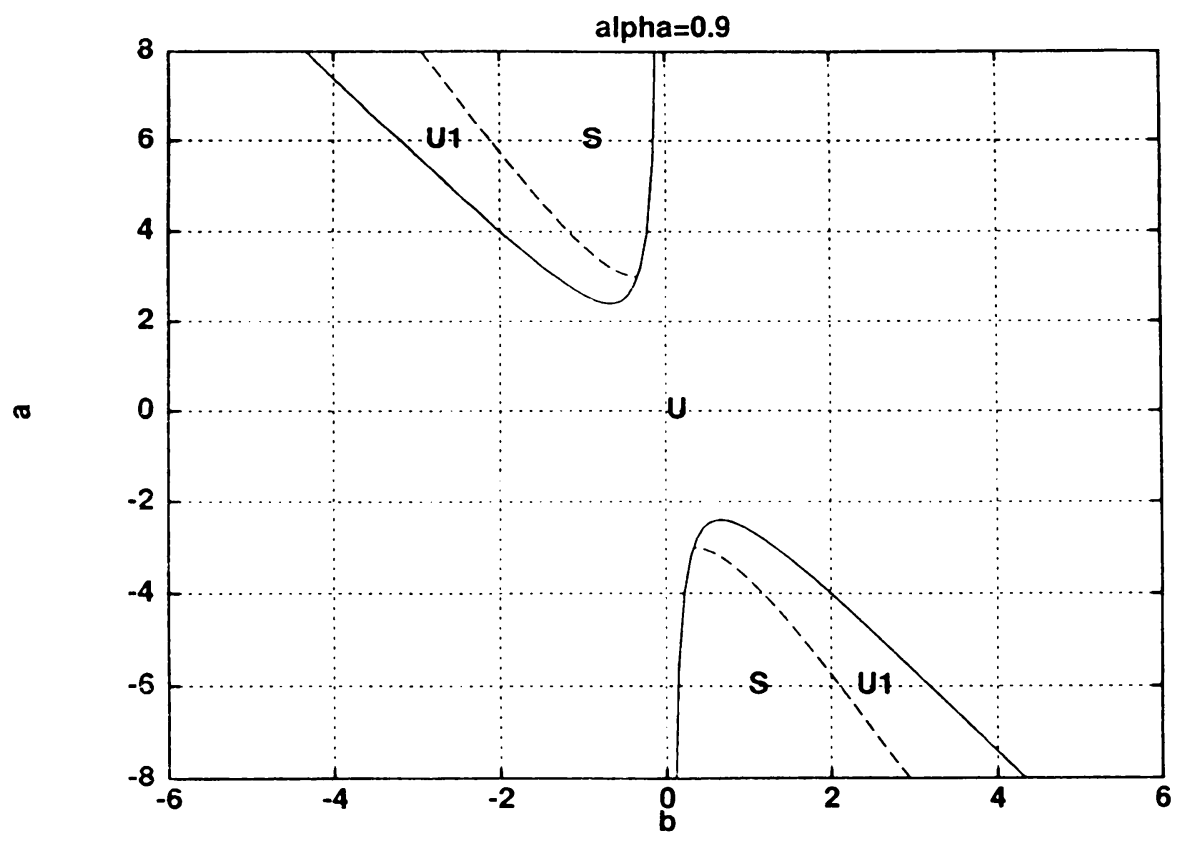

FIG. 3(e). Region of FW stability in the $(b, a)$ plane for a single complex Ginzburg-Landau equation; $\alpha=0.9$. 


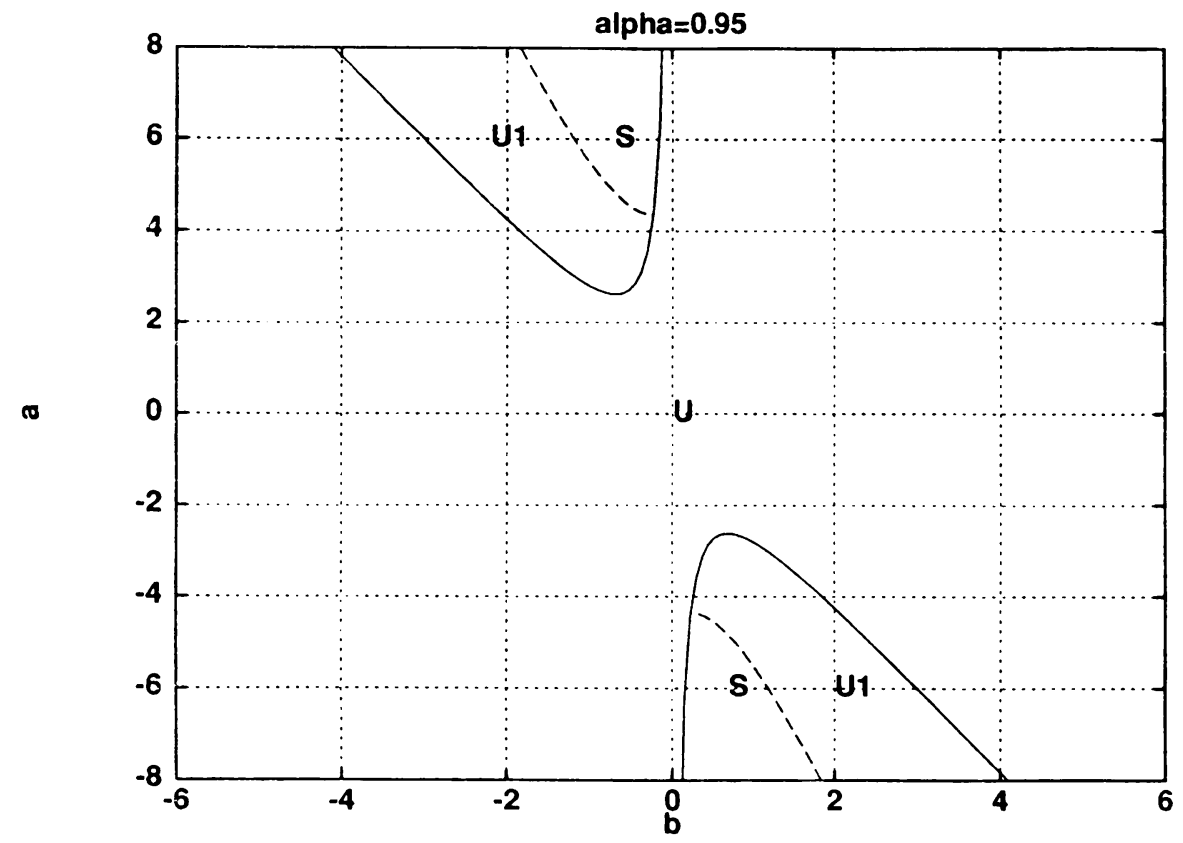

FIG. 3(f). Region of FW stability in the $(b, a)$ plane for a single complex Ginzburg-Landau equation; $\alpha=0.95$.

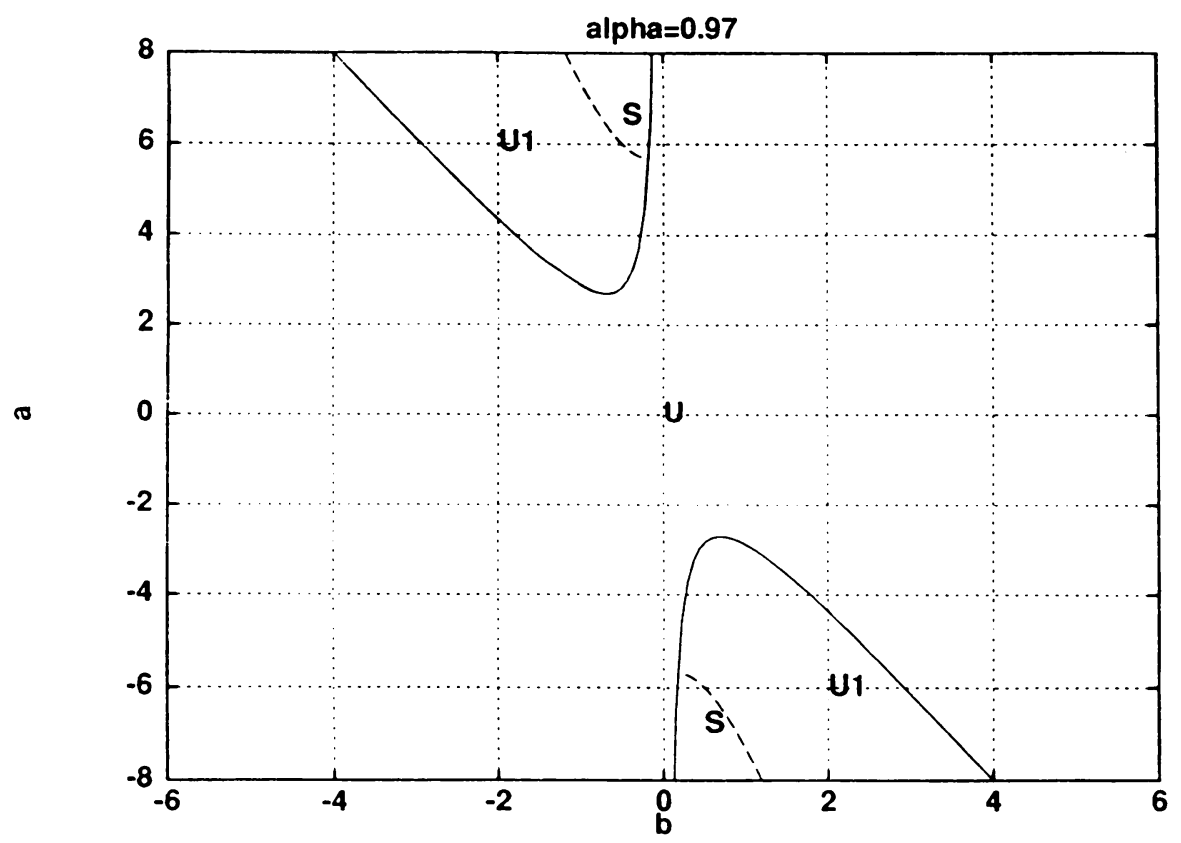

FIG. 3(g). Region of FW stability in the $(b, a)$ plane for a single complex Ginzburg-Landau equation; $\alpha=0.97$. 


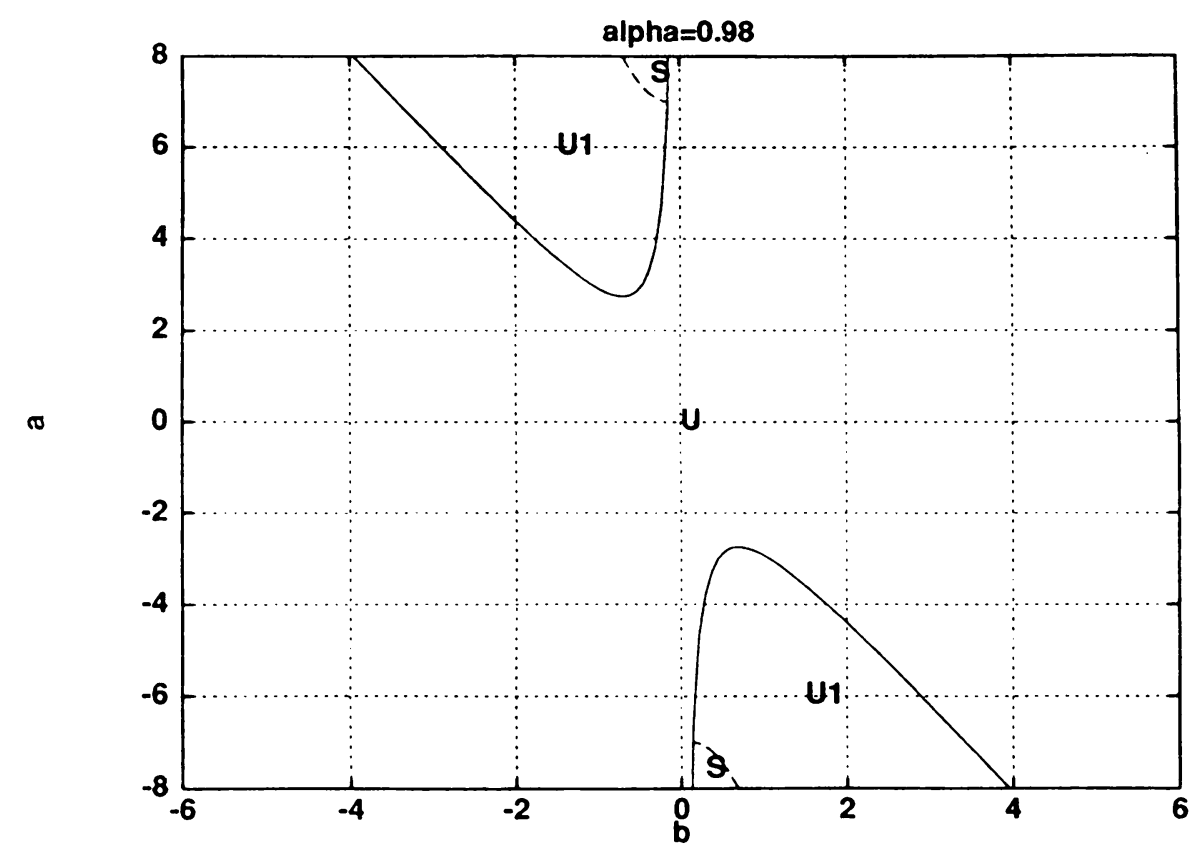

FIG. 3(h). Region of FW stability in the $(b, a)$ plane for a single complex Ginzburg-Landau equation; $\alpha=0.98$.

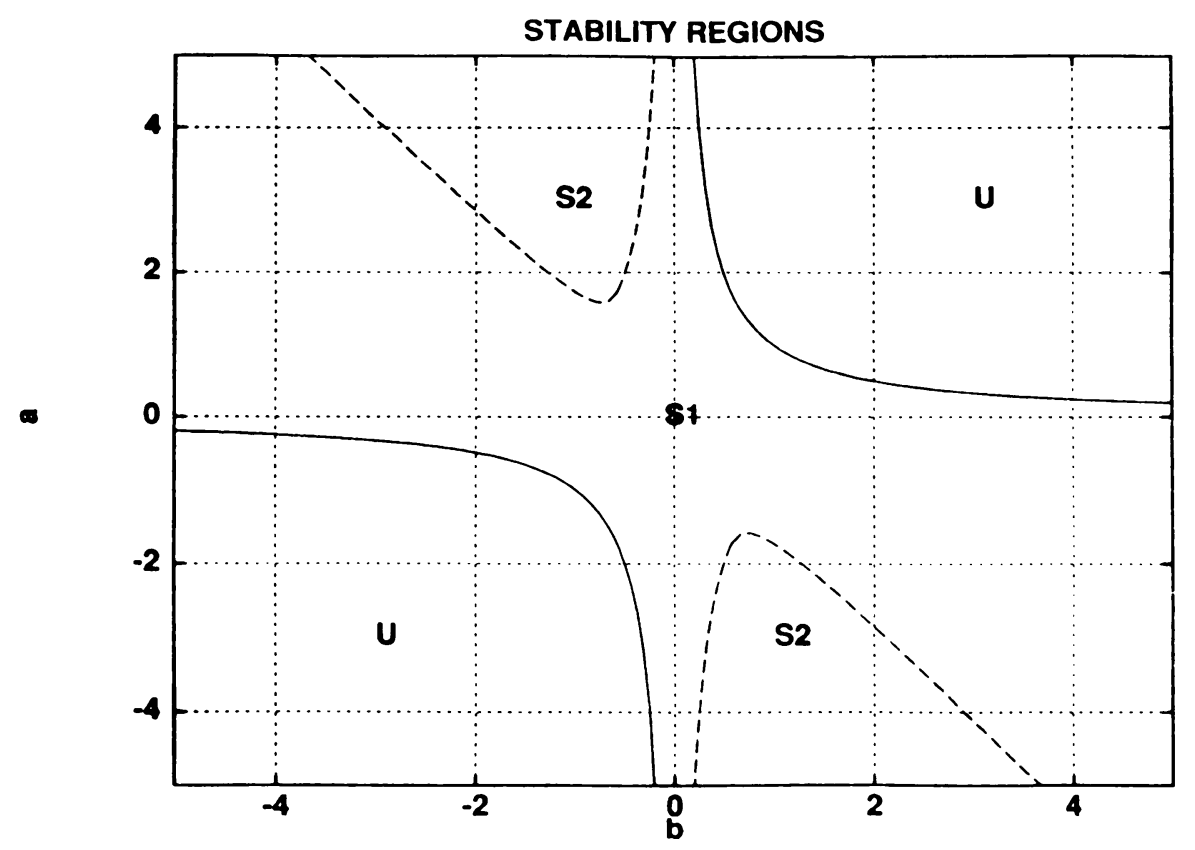

FIG. 4. Regions of stability in the $(b, a)$ plane for a single complex Ginzburg-Landau equation. 


\section{REFERENCES}

[1] W. Eckhaus, Studies in Nonlinear Stability Theory, Springer, Berlin, 1965

[2] G. B. Ermentrout, Stable small-amplitude solutions in reaction-diffusion systems, Quart. Appl. Math. 39, 61-86 (1981)

[3] L. N. Howard and N. Kopell, Slowly varying waves and shock structures in reaction-diffusion equations, Stud. Appl. Math. 56, 95-145 (1977)

[4] E. Knobloch and J. DeLuca, Amplitude equations for travelling wave convection, Nonlinearity 3, 975-980 (1990)

[5] L. R. Keefe, Dynamics of perturbed wavetrain solutions to the Ginzburg-Landau equation, Stud. Appl. Math. 73, 91-153 (1985)

[6] Y. Kuramoto, Chemical Oscillations, Waves and Turbulence, vol. 19, Springer Series in Synergetics, Springer-Verlag, Berlin and New York, 1984

[7] Y. Kuramoto and S. Koga, Anomalous period-doubling bifurcations leading to chemical turbulence, Phys. Lett. 92A, 1-4 (1982)

[8] C. G. Lange and A. C. Newell, A stability criterion for envelope equations, SIAM J. Appl. Math. 27, 441-456 (1974)

[9] B. J. Matkowsky and V. Volpert, Coupled nonlocal complex Ginzburg-Landau equations in gasless combustion, Physica 54D, 203-219 (1992)

[10] H. T. Moon, P. Huerre, and L. G. Redekopp, Transitions to chaos in the Ginzburg-Landau equation, Physica 7D, 135-150 (1983)

[11] A. C. Newell, Dynamics of patterns: A survey, Propagation in Systems Far from Equilibrium, Proceedings of Les Houches Workshop (J. E. Wesfried, H. R. Brand, P. Manneville, G. Albinet, and N. Boccara, eds.), Springer-Verlag, Berlin, Heidelberg, 122-155, 1987

[12] A. C. Newell and J. A. Whitehead, Finite bandwidth, finite amplitude convection, J. Fluid Mech. 38, 279-303 (1969)

[13] P. K. Newton and L. Sirovich, Instabilities of the Ginzburg-Landau equation: periodic solutions, Quart. Appl. Math. 44, 49-58 (1986)

[14] P. K. Newton and L. Sirovich, Instabilities of the Ginzburg-Landau equation: Part II. Secondary bifurcation, Quart. Appl. Math 44, 367-374 (1986)

[15] K. Nozaki and N. Bekki, Pattern selection and spatiotemporal transition to chaos in the GinzburgLandau equation, Phys. Rev. Lett. 51, No. 24, 2171-2174 (1983)

[16] D. O. Olagunju and B. J. Matkowsky, Burner stabilized cellular flames, Quart. Appl. Math. 48, 645-664 (1990)

[17] D. O. Olagunju and B. J. Matkowsky, Coupled complex Ginzburg-Landau type equations in gaseous combustion, Stability and Applied Analysis of Continuous Media 2, No. 1, 1-27 (1992)

[18] J. D. Rodriguez and L. Sirovich, Low dimensional dynamics for the complex Ginzburg-Landau equation, Physica 43D, 77-86 (1990)

[19] W. Schöpf and L. Kramer, Small-amplitude periodic and chaotic solutions of the complex GinzburgLandau equation for a subcritical bifurcation, Phys. Rev. Lett. 66, 2316-2319 (1991)

[20] L. A. Segel, Distant side-walls cause slow amplitude modulation of cellular convection, J. Fluid Mech. 38, 203-224 (1969)

[21] L. Sirovich and P. K. Newton, Periodic solutions of the Ginzburg-Landau equation, Physica 21D, 115-125 (1986)

[22] L. Sirovich, J. D. Rodriguez, and B. Knight, Two boundary value problems for the Ginzburg-Landau equation, Physica 43D, 63-76 (1990)

[23] K. Stewartson and J. T. Stuart, A non-linear instability theory for a wave system in plane Poiseuille flow, J. Fluid Mech. 48, 529-545 (1971)

[24] J. T. Stuart and R. C. DiPrima, The Eckhaus and Benjamin-Feir resonance mechanisms, Proc. Roy. Soc. London Ser. A 362, 27-41 (1978) 